\title{
OGLE-2016-BLG-0168 Binary Microlensing Event: Prediction and Confirmation of the Microlens Parallax Effect from Space-based Observations
}

\author{
I.-G. Shin ${ }^{1,16}$ (1) , A. Udalski ${ }^{2,17}$, J. C. Yee ${ }^{1,16,18}$ (), S. Calchi Novati ${ }^{3,4,18}$ (1D, C. Han ${ }^{5}$, \\ and \\ J. Skowron ${ }^{2}$ (D), P. Mróz ${ }^{2}$, I. Soszyński ${ }^{2}$, R. Poleski ${ }^{2,6}$, M. K. Szymański², S. Kozłowski² (iD, P. Pietrukowicz ${ }^{2}$ (D), K. Ulaczyk ${ }^{2,7}$, \\ M. Pawlak ${ }^{2}$ \\ (OGLE Collaboration),

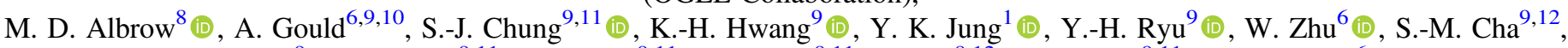

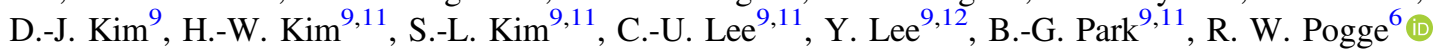 \\ (KMTNet Group), \\ and \\ C. Beichman ${ }^{13}$, G. Bryden ${ }^{14}$, S. Carey ${ }^{15}$ (D) B. S. Gaudi ${ }^{6}$ (D) C. B. Henderson ${ }^{14,19}$ (D), and Y. Shvartzvald ${ }^{14,19}$ (D) \\ (Spitzer Team)

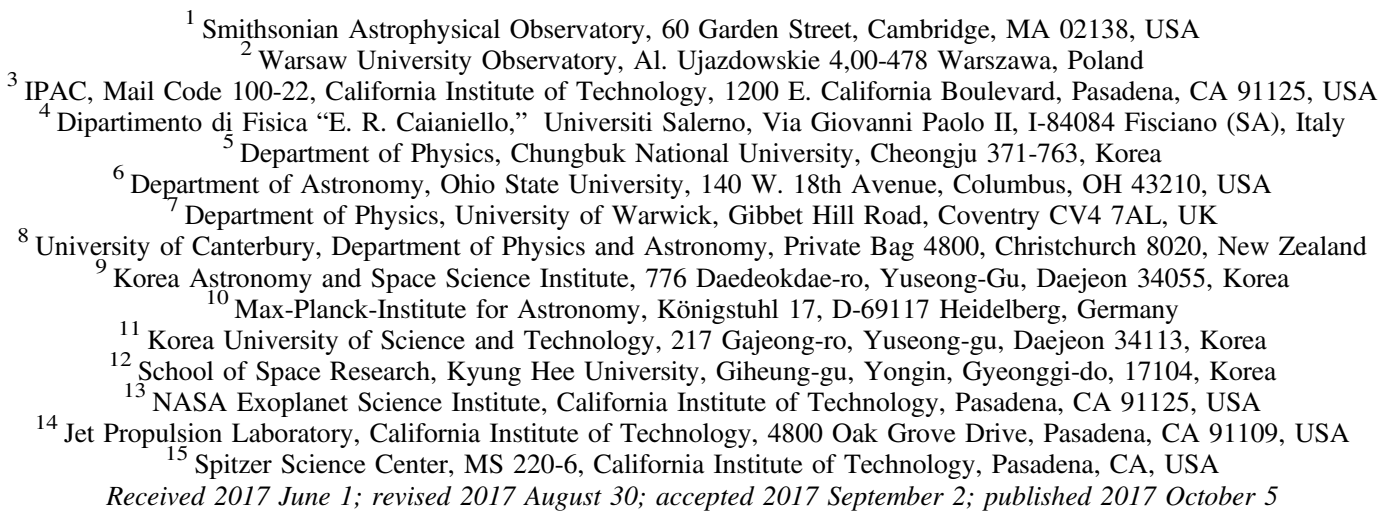

\section{Abstract}

The microlens parallax is a crucial observable for conclusively identifying the nature of lens systems in microlensing events containing or composed of faint (even dark) astronomical objects such as planets, neutron stars, brown dwarfs, and black holes. With the commencement of a new era of microlensing in collaboration with space-based observations, the microlens parallax can be routinely measured. In addition, space-based observations can provide opportunities to verify the microlens parallax measured from ground-only observations and to find a unique solution to the lensing light-curve analysis. Furthermore, since most space-based observations cannot cover the full light curves of lensing events, it is also necessary to verify the reliability of the information extracted from fragmentary space-based light curves. We conduct a test based on the microlensing event OGLE-2016-BLG-0168, created by a binary lens system consisting of almost equal mass M-dwarf stars, to demonstrate that it is possible to verify the microlens parallax and to resolve degeneracies using the space-based light curve even though the observations are fragmentary. Since space-based observatories will frequently produce fragmentary light curves due to their short observing windows, the methodology of this test will be useful for next-generation microlensing experiments that combine space-based and ground-based collaboration.

Key words: binaries: general - gravitational lensing: micro

\section{Introduction}

The microlensing technique can probe a variety of astronomical objects in a wide range of masses such as planets, neutron stars, brown dwarfs, and isolated black holes (Poindexter et al. 2005; Dong et al. 2007; Miyake et al. 2012; Shvartzvald et al. 2015; Wyrzykowski et al. 2016). The microlensing technique can detect these faint or dark objects

\footnotetext{
${ }_{17}^{16}$ KMTNet Group.

17 OGLE Collaboration.

18 Spitzer Team.

19 NASA Postdoctoral Program Fellow.
}

regardless of their luminosity levels, in sharp contrast to other methods, which as a matter of course are restricted to studying objects within their flux detection limits.

Microlensing surveys are primarily focused on finding planets and characterizing planetary populations, including planets in binary star systems. Therefore, one significant purpose of conducting detailed analyses of binary microlensing events is to probe the potential existence of planets such as companions to two-body systems. To find subtle deviations caused by planets, a precise and reliable model of the binary lensing light curve is required. Then, even if an absence of planets is observed, as in this event, an analysis of the planet 
sensitivity can provide constraints on planet frequencies for various host stars in our galaxy. Such an analysis is beyond the scope of this paper, but a key element of understanding planets in binary systems is to first reveal the lens properties based on modeling of the binary light curve. Furthermore, it is necessary to demonstrate that the observed microlensing effects contributing to the physical characterization of the binary are reliably measured.

To conclusively reveal the nature of the lens system that generates a microlensing event, additional observables are required, such as the microlens parallax, $\pi_{\mathrm{E}}$, and the angular Einstein ring radius, $\theta_{\mathrm{E}}$. Based on these additional observables, the properties of the lens system can be determined from

$$
M_{\mathrm{L}}=\frac{\theta_{\mathrm{E}}}{\kappa \pi_{\mathrm{E}}} ; \quad D_{\mathrm{L}}=\frac{\mathrm{au}}{\pi_{\mathrm{E}} \theta_{\mathrm{E}}+\pi_{\mathrm{S}}},
$$

where $M_{\mathrm{L}}$ is the total mass of the lens system, $D_{\mathrm{L}}$ is the distance to the lens system toward the Galactic bulge, $\pi_{\mathrm{S}}$ is the parallax of the background star (source) defined as $\pi_{\mathrm{S}}=\mathrm{au} / D_{\mathrm{S}}$, where $D_{\mathrm{S}}$ is the distance to the source, and $\kappa \equiv 4 G /\left(c^{2} \mathrm{au}\right) \sim 8.14 \mathrm{mas} / M_{\odot}$. Although $\pi_{\mathrm{E}}$ and $\theta_{\mathrm{E}}$ appear equally important in Equation (1), $\pi_{\mathrm{E}}$ is actually more crucial because $\theta_{\mathrm{E}}$ is easily determined from the finite source effect with high-cadence observations. In particular, for a binary lensing event, $\theta_{\mathrm{E}}$ can be routinely measured when the source crosses or approaches caustics of binary lensing events. Thus, it is important to securely and accurately measure the microlens parallax.

However, measurement of the microlens parallax based on ground-only observations is made from subtle deviations in those lensing light curves that have a sufficiently long timescale to make manifest the deviations caused by Earth's orbital motion. As a result, there exist some obstacles to measuring the microlens parallax. First, the signal of the microlens parallax, i.e., subtle deviations in the light curve, can be detected if Earth moves enough to produce the signal over the duration of the event. Thus, the microlens parallax can be measured for only some cases of lensing events that have long timescales (usually, $t_{\mathrm{E}} \geqslant 30$ days). Second, the subtle deviations in the light curve due to microlens parallax effects can be confused with photometric systematics that can lead to a false positive detection or inaccurate measurement of the annual microlens parallax. For example, systematics in MOA data, which are taken under difficult conditions, occasionally lead to spurious detections of the microlens parallax that are contradicted by other data sets (e.g., Hirao et al. 2017). Therefore, it is important to verify, where possible, that the annual microlens parallax effect has been accurately measured. Third, there exist degeneracies in the microlens parallax that prevent accurately or uniquely measuring it. For example, the ecliptic degeneracy (Jiang et al. 2004; Skowron et al. 2011) produces degenerate solutions with different values of the microlens parallax that can describe the same lensing light curve. Also, the lens-orbital effect caused by the orbital motion of the lens components affects the measured values of the microlens parallax (Batista et al. 2011; Skowron et al. 2011; Shin et al. 2012). Hence, before the era of spacebased microlensing, the microlens parallax could be securely and accurately measured for only a small number of lensing events that satisfy conditions to measure it during a bulge season.

In the new era, however, the microlens parallax can be routinely and securely measured in collaboration with space-based observations. In principle, the offset between ground and space telescopes provides a chance to routinely measure the microlens parallax regardless of the magnification level of the lensing event. In addition, space-based observations can provide opportunities to verify the measurement of the microlens parallax and to resolve degeneracies in the microlens parallax.

When space-based observations cover the same features seen with the ground-based light curve, the measurement of the microlens parallax is straightforward (Udalski et al. 2015b). However, if only one caustic crossing for a binary is seen, this can lead to significant degeneracies in the measurement of the microlens parallax (Zhu et al. 2015). For lensing events with relatively long timescales, space-based observations can cover only fragmentary parts of the full lensing light curve due to short observing windows, so it is possible no caustic crossings will be observed because no caustic crossings occur during that window (as in this case). For example, the Spitzer Space Telescope has only a $\sim 40$ day observing window. Furthermore, there is a 3-10 day delay between selecting a target for Spitzer observations and the start of those observations, which may further decrease the size of the observing window. As a result, we frequently must use fragmentary space-based observations without caustic-crossing features to measure the microlens parallax. In fact, during the Spitzer microlensing campaign in 2015-2016, most of the observed light curves are fragmentary. Thus, it is important to perform a test to see whether it is possible to extract reliable information from them or not.

For single-lensing events, Yee et al. (2015a) posit and Calchi Novati et al. (2015a) and Zhu et al. (2017) show that fragmentary light curves can be successfully exploited to extract microlens parallaxes for point-lens (or near point-lens) events. However, there exist only a few cases that have demonstrated that microlens parallaxes can be retrieved for binary lensing events with fragmentary space-based light curves without caustic-crossing features (Han et al. 2017; Shvartzvald et al. 2017). Indeed, Han et al. (2017) used fragmentary space-based observations obtained from Spitzer to show that the microlens parallax was measured and to confirm the measurement based on the annual microlens parallax effect.

We conduct another such test using the binary microlensing event OGLE-2016-BLG-0168, which has Spitzer observations. The event has a long timescale $\left(t_{\mathrm{E}} \sim 90\right.$ days) and the Spitzer observations covered a short part ( $\sim 30$ days) of the full lensing light curve. Moreover, we found degenerate solutions to the event during the analysis. As a result, this event is a perfect testbed to show the possibility of extracting information from the fragmentary light curve observed by a space-based observatory. Our test can provide an important example for probing the reliability of extracting information from the fragmentary spacebased observations. In addition, the methodology of this test can provide procedures to systematically measure and verify the microlens parallax based on fragmentary light curves from space and to resolve the degenerate solutions, especially for the Spitzer microlensing campaign. Finally, this event can verify that the annual microlens parallax can be securely measured from ground-based data even in the presence of systematics, regardless of the source of those systematics.

In this paper, we describe observations of the event in Section 2. In Section 3, we describe our analysis procedures and the test. In Section 4, we present results of the analysis and the test of the event. Lastly, we discuss and summarize the results in Section 5. 

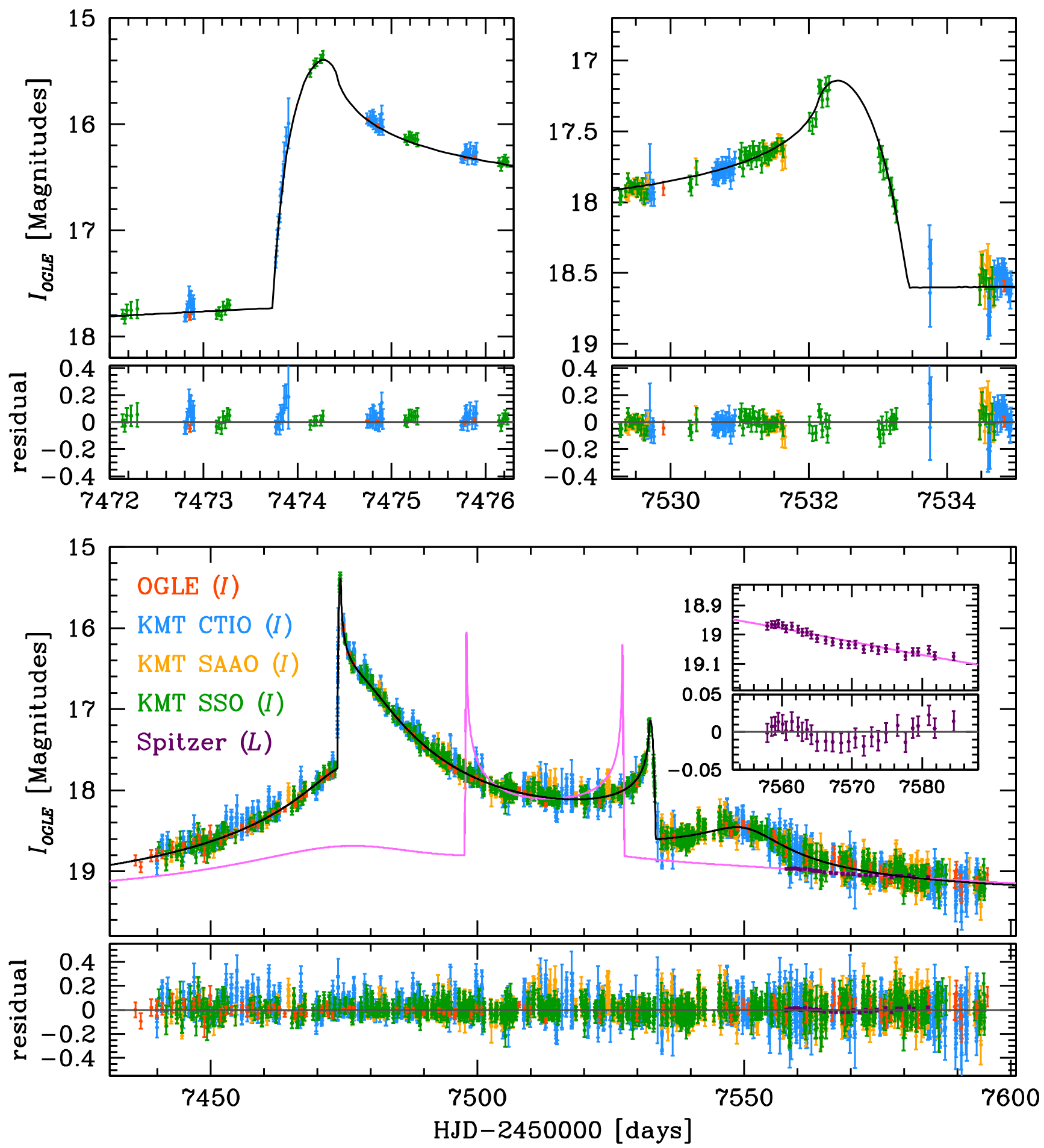

Figure 1. Light curves of the binary microlensing event OGLE-2016-BLG-0168. Each color represents observed data from different telescopes located on the ground and in space. The black and pink solid lines indicate the model light curves of ground and Spitzer observations, respectively. The upper panels show the zoom-ins of the ground light curves for the caustic entrance (left) and exit (right). The lower panels show the whole light curves with residuals between models and observations; for clarity, the inset shows just the Spitzer data.

\section{Observations}

The microlensing event OGLE-2016-BLG-0168 occurred on the source star located in the galactic bulge at $(\alpha, \delta)_{\mathrm{J} 2000}=\left(17^{\mathrm{h}} 50^{\mathrm{m}} 49.89,-31^{\circ} 45^{\prime} 300^{\prime \prime} 1\right)$ in equatorial coordinates and $(l, b)=(-1.84,-2.42)$ in galactic coordinates. The event was observed both by ground-based surveys and by
Spitzer. In Figure 1, we present the observed light curve of OGLE-2016-BLG-0168. The upper two panels show the caustic-crossing parts of the light curve and the lower panels show the entire duration of significant magnification. The light curve observed from ground-based telescopes shows typical features caused by a binary lens system. 


\subsection{Ground-based Observations}

The event was announced by the Optical Gravitational Lensing Experiment (OGLE: Udalski et al. 2015a) based on observations with its $1.3 \mathrm{~m}$ Warsaw telescope with a $1.4 \mathrm{deg}^{2}$ camera located at the Las Campanas Observatory in Chile. The event was alerted by the Early Warning System (Udalski et al. 1994; Udalski 2003) of the OGLE survey on 2016 February 21. The OGLE data in the $I$-band were reduced by a pipeline based on the difference-imaging analysis method (Alard \& Lupton 1998; Wozniak 2000). The uncertainties of the OGLE data were re-scaled according to the description in Skowron et al. (2016).

The Korea Microlensing Telescope Network (KMTNet: Kim et al. 2016) survey, which is designed for high-cadence monitoring toward the galactic bulge with a large field-of-view, independently observed the event. KMTNet is a telescope network consisting of three identical $1.6 \mathrm{~m}$ telescopes with $4 \mathrm{deg}^{2}$ cameras located at the Cerro Tololo Inter-American Observatory in Chile (KMTC), the South African Astronomical Observatory in South Africa (KMTS), and the Siding Spring Observatory in Australia (KMTA). For the event, KMTC and KMTA observations covered the caustic entrance $\left(\mathrm{HJD}-2450000=\mathrm{HJD}^{\prime} \sim 7474.2\right)$ and exit $\left(\mathrm{HJD}^{\prime} \sim\right.$ 7532.5) parts of the light curve with a $\sim 15$ minute cadence. KMTNet data in the I-band were reduced by pySIS (Albrow et al. 2009), which employs the image subtraction method.

The event was also observed in both the $I$ and $H$ bands by the SMARTS $1.3 \mathrm{~m}$ telescope at CTIO in Chile. These data were not used in the modeling, but were used to determine the $(I-H)$ source color (see Section 3.3).

\subsection{Space-based Observations}

The event was observed by the Spitzer space telescope with the $3.6 \mu \mathrm{m}$ channel (hereafter, $L$-band) of the IRAC camera. Briefly, the event was selected on 2016 June 16 as a subjective target based on the selection criteria described in Yee et al. (2015a) because the light curve from ground-based observations showed typical anomaly features caused by the binary lens system. The observations started on 2016 June 18 $\left(\mathrm{HJD}^{\prime} \sim 7557.93\right)$ and ended July 14 (HJD $\left.\sim 7584.48\right)$. During 4 weeks of observations with cadence $\sim 1$ day $^{-1}, 28$ data points for the event were gathered and then the data were reduced using methods described in Calchi Novati et al. (2015b). In the Spitzer observations, there exist sinusoidal trends whose origins are unknown. However, we can securely determine the modeling parameters in this case despite the trend in the Spitzer data. Indeed, several publications used Spitzer data (which also had similar photometric systematics) to conclude that the systematics in Spitzer data are not likely to be an issue for determining modeling parameters (e.g., Poleski et al. 2016; Shvartzvald et al. 2017; Zhu et al. 2017).

\subsection{Extinction}

The source star of the event is located in a severely extincted field. The $A_{I}$ value is measured from the color-magnitude diagram (CMD) analysis of this event (see Section 3.3). Based on the $I$-band extinction, the $A_{L}$ value is calculated using the relationship between optical and infrared extinction (Cardelli et al. 1989). The source extinction is $A_{I} \sim 4.9$ in the $I$-band and $A_{L} \sim 0.35$ in the $L$-band. As a result, the source is relatively faint for ground-based observations from OGLE and
KMTNet. In contrast to ground-based observations, the source is a quite bright target for Spitzer observations.

\section{Analysis}

We model the light curves of the OGLE-2016-BLG-0168 event to reveal the nature of the binary lens system causing the microlensing event. In addition, we conduct a test to validate the microlens parallax and resolve the degeneracy in the microlens parallax.

Because the event was simultaneously observed by ground and space telescopes, we try to find fits for both observed light curves using parameters adopted from the conventional parameterization (Refsdal 1966; Gould 1992, 1994; Graff \& Gould 2002; Shin et al. 2013; Jung et al. 2015; Udalski et al. 2015b; Zhu et al. 2015). We briefly summarize the parameterization to facilitate further description of the modeling. In total, we used 11 geometric parameters to construct model light curves considering the higher-order effects. Among these, seven parameters $\left(t_{0}, u_{0}, t_{\mathrm{E}}, s, q, \alpha\right.$, and $\left.\rho_{*}\right)$ were used to describe the static binary lens model. The other four parameters were used to describe the vector $\pi_{\mathrm{E}}$ components $\left(\pi_{\mathrm{E}, N}, \pi_{\mathrm{E}, E}\right)$ of the microlens parallax and orbital motion $(d s / d t, d \alpha / d t)$ of the binary lens components. For the parameters of the static binary lens model, $t_{0}, u_{0}, t_{\mathrm{E}}$, and $\alpha$ are related to descriptions of the trajectory of the magnified background star (hereafter, source) as seen from the ground, which are defined as the time of the closest source approach to the center of mass of the binary lens system, the impact parameter (separation between the center of mass and the source position at time of $t_{0}$ ), the source crossing time along the angular Einstein ring radius, i.e., $\theta_{\mathrm{E}}$, and the angle of the source trajectory with respect to the binary axis, respectively. The parameters $s$ and $q$ are related to describing the caustic structure and are defined as the projected separation between the binary stars normalized by $\theta_{\mathrm{E}}$ and the mass ratio of the primary and secondary stars, respectively. The last parameter $\rho_{*}$ is defined as the source radius normalized by $\theta_{\mathrm{E}}$, i.e., $\rho_{*}=\theta_{*} / \theta_{\mathrm{E}}$, which can provide a measurement of $\theta_{\mathrm{E}}$ based on the finite source effect that moderates the amplitude of magnification when the source crosses the caustics.

The modeling consists of three phases. In the first phase, to find a global minimum, we conduct a grid search of the $(s, q)$ parameter space with those variables fixed, because the parameters are directly related to the caustic structure, which leads to dramatic changes in the features of the static binary model light curve. In this phase, we consider static two-body models for the grid search. The boundaries of the grid ranges are set as $\log (s)=[-1.0,1.0]$ and $\log (q)=[-5.0,1.0]$ and each range is divided by 100 . In addition, the model light curves are also sensitive to $\alpha$. Thus, $\alpha$ is treated as a "semigrid" parameter that can provide various initial values for the grid search. This parameter starts from 21 grid points that will be initial values within a whole range of $[0,2 \pi](\mathrm{rad})$, and then the $\alpha$ parameter is treated as a free parameter that can be varied within the range. As a result, the total number of grid points is $100 \times 100 \times 21$. This set of grid points is enough to find all local minima for binary lensing cases including planetary cases. For the other four basic parameters, we allow these parameters to be varied within the full ranges to cover all possible cases. The initial values of these parameters are defined from the point-lens fitting without binary lensing perturbations, except the $\rho_{*}$ parameter. For the $\rho_{*}$ parameter, the initial value is set to 
a typical angular source radius normalized by the Einstein ring radius based on the baseline magnitude of the lensing event. The values are set to 0.03 or 0.003 for cases of giant or dwarf source stars in the galactic bulge, respectively. For the $\chi^{2}$ minimization method, we use a Markov Chain Monte Carlo (MCMC) algorithm. The parameters are varied, with proper steps defined by covariance vectors from the initial values.

In the second phase, based on the best static binary model found in the first phase, we sequentially introduce the higherorder effects caused by the microlens parallax and the orbital motion of the binary lens components. These effects can produce better fits if there exist remaining residuals between the static model and the observed light curve. Note that both effects should be simultaneously considered because both simultaneously affect the curvature of the source trajectory and reflect physical motions. In this phase, we treat all parameters, including parameters describing the higher-order effects, as free parameters that can be varied within the full ranges.

In the last phase, we refine the models after re-scaling the errors of the observed data based on the best-fit model, so that each data point can be represented as $\sim 1 \chi^{2} /$ dof when the models are computed. This re-scaling process is essential because the original instrumental magnitude and error of individual data sets were measured by different photometry tools developed by each survey group, i.e., individual data sets have different zero-points and error bars scaled by different methods. As a result, one original data point cannot represent $\chi^{2} \sim 1$. Hence, it requires aligning data sets and re-scaling their errors for a meaningful comparison between $\chi^{2}$ values. For this, we aligned all data sets to the OGLE observations and then re-scaled their error bars based on the best-fit model. As a result, each data point contributes $\Delta \chi^{2} \sim 1$, as expected for Gaussian noise.

During the refining process, we consider the variation of the magnification due to the limb-darkening of the source's surface by adopting coefficients from Claret (2000) that correspond to the source type of the event (in Section 3.3, determining the source type is described in detail). In this phase, we estimate uncertainties of model parameters based on the scatter of each MCMC chain.

\subsection{Modeling of the Ground-based Light Curve}

In Figure 1, we present observed light curves as seen from ground and space. The light curve shows a typical " $U$ "-shape of a binary lensing light curve. As shown in the zoom-ins, the caustic entrance and exit are well-covered by the KMTNet survey, thus we can clearly measure the angular Einstein ring radius. In addition, the time between the caustic entrance and exit is $\sim 60$ days. This is long enough to expect to detect signals in the ground-based light curve caused by the annual microlens parallax and lens-orbital effects. In Figure 2, we also present the geometry of the event, which is described in detail in Sections 4.1 and 4.3 .

In Table 1, we present models with the best-fit parameters of the various solutions considering the lens-parallax and lensorbital effects. We find that there exist two degenerate models, $\left(u_{0}<0\right)$ and $\left(u_{0}>0\right)$, for the ground-based light curve. For both cases, we consider the microlens parallax and the lensorbital effects. When the microlens parallax effect (annual microlens parallax effect: Gould 1992) is introduced, we find that additional $\chi^{2}$ improvements compared to the static model are 159.4 and 152.6 for the $\left(u_{0}<0\right)$ and $\left(u_{0}>0\right)$ cases, respectively. In addition, when we supplement the lens-parallax model with the lens-orbital effect (approximated lens-orbital effect: Shin et al. 2013), we find that the $\chi^{2}$ improvements are 115.0 and 108.7 for the $\left(u_{0}<0\right)$ and $\left(u_{0}>0\right)$ cases, respectively. This implies that both effects are clearly detected for both cases. Since we found significant $\chi^{2}$ improvement when we considered the lens-orbital effect, we investigated complete Keplerian orbital solutions (with parameters adopted from Shin et al. 2011; Skowron et al. 2011). However, the Keplerian orbital solutions could not be meaningfully constrained for this case. Hence, in this work, we adopt the approximated lens-orbital parameters when we consider the lens-orbital effect for the best-fit models.

For the ground-based models considering the microlens parallax and lens-orbital effects, the $\chi^{2}$ difference between the best-fit models in Table 1 is only $\chi^{2}\left(u_{0}>0\right)-\chi^{2}\left(u_{0}<0\right)=13.4$. Hence, the $\left(u_{0} 0<0\right)$ solution is slightly preferred. We note that for this event there do not exist degenerate solutions caused by the close/wide degeneracy (Griest \& Safizadeh 1998; Dominik 1999; An 2005) because the best-fit models have resonant caustics $(s \sim 1)$.

There exist some residuals to the caustic entrance part of the light curve $\left(\mathrm{HJD}^{\prime} \sim 7473.9\right)$. These short-timescale systematics ( $\lesssim 1$ day timescales) are not expected to affect the determination of the microlensing parameters for such a longtimescale event $\left(t_{\mathrm{E}} \gg 1\right.$ day). Photometric systematics affecting the determination of the microlens parallax or lens-orbital motion parameters typically occur in the baseline data of an event and relate to long-timescale variations, i.e., systematics with timescales $\sim t_{\mathrm{E}}$ (Smith et al. 2003).

These short-timescale systematics might affect the determination of these parameters if they contributed significant uncertainty to the time of the caustic entrance, which serves as a strong, fixed reference point for the determination of the lensorbital parameters. However, despite the systematics in the KMTC data, the time of the caustic entrance is extremely well defined, especially compared to the $\sim 60$-day timescale between the entrance and exit. In addition, it is possible to securely measure the angular source radius, i.e., $\rho_{*}$, from the caustic exit part of the light curve. Thus, any uncertainties in this parameter due to systematics in the caustic entrance are more than compensated for by other data. Furthermore, this measurement of $\rho_{*}$ means that $t_{*}=\rho_{*} / t_{\mathrm{E}}$ is well determined, such that given the KMTA data over the peak of the caustic entrance, the timing of that caustic entrance is fixed independent of the KMTC data. Therefore, the small systematics seen in the KMTC data the night of $\sim 7473.9$ have no influence on the model parameters. We have confirmed this by repeating the modeling excluding those data.

\subsection{Modeling of the Space-based Light Curve}

We conduct modeling of the combined ground and Spitzer observations. We present observed light curves with the best-fit models in Figure 1. During the modeling process, we investigated degenerate solutions caused by the "four-fold degeneracy" (Zhu et al. 2015). The degeneracies are caused by different source trajectories seen by ground and space telescopes passing over a similar lensing magnification pattern, which is reflected over the binary axis. The degenerate solutions are denoted by the combination of the signs of impact parameters 

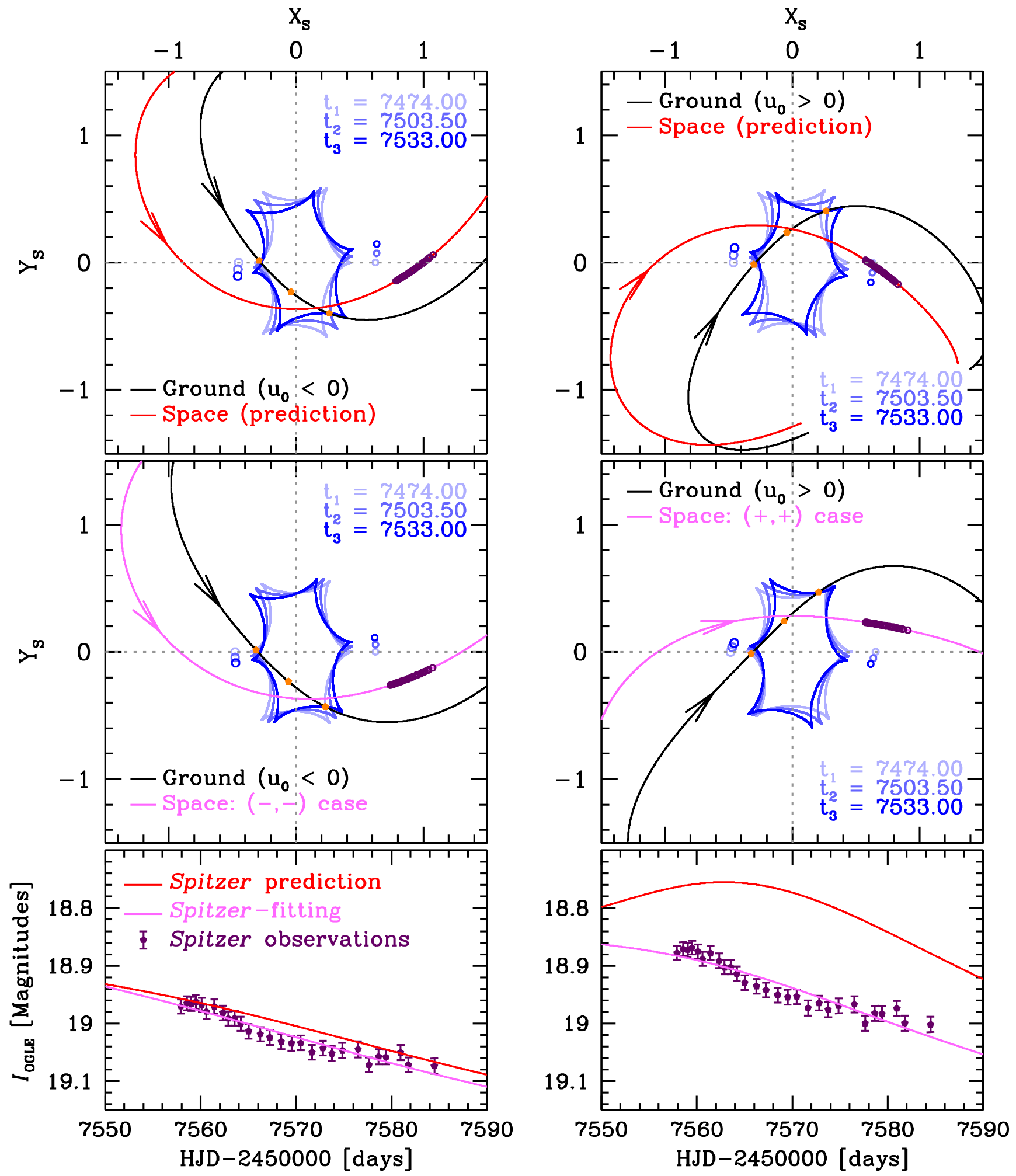

Figure 2. Geometries of the binary microlensing event OGLE-2016-BLG-0168. The top panels show caustic features reflecting the orbital motion of the binary lens system at the time when the source enters $\left(\mathrm{HJD}^{\prime} \sim 7474.0\right)$, is inside $(\sim 7503.5)$, and exits $(\sim 7533.0)$ the caustic; the orange dots mark the source position at those times. In the panels, the black line with an arrow indicates the source trajectory of ground-based models and the red line indicates the predicted source trajectory of the Spitzer light curve based on the ground models of the $\left(u_{0}<0\right)$ (left) and $\left(u_{0}>0\right)$ (right) cases. The purple points represent the coverage of Spitzer observations. The middle panels show the geometries of models with combined data from the ground and Spitzer. The color scheme is the same as the top panel, except now a pink line indicates the Spitzer-fitted light curve. The bottom panels show the prediction, Spitzer-fitting, and observations of the Spitzer light curve for both $(-,-)$ (left) and $(+,+)$ (right) cases, respectively.

as seen from the space and ground, i.e., $( \pm, \pm)$, according to convention (see Section 3 in Zhu et al. 2015). Under our parameterization, we can control the source trajectories by changing the sign of $u_{0}$ and the $\pi_{\mathrm{E}, N}$ parameters and thus we carefully set initial values to search for these models. For this event, we find that the $(-,-)$ and $(+,+)$ models showed similar 
Table 1

The Best-fit Models of the Ground-based Observations

\begin{tabular}{|c|c|c|c|c|c|}
\hline \multirow[b]{2}{*}{ Parameter } & \multirow[b]{2}{*}{ STD } & \multicolumn{2}{|c|}{$\left(u_{0}<0\right)$} & \multicolumn{2}{|c|}{$\left(u_{0}>0\right)$} \\
\hline & & PRX & $\mathrm{OBT}+\mathrm{PRX}$ & PRX & OBT+PRX \\
\hline$\overline{\chi^{2} / \mathrm{dof}}$ & $6501.53 /(6232-7)$ & $6342.13 /(6232-9)$ & $6227.09 /(6232-11)$ & $6348.94 /(6232-9)$ & $6240.25 /(6232-11)$ \\
\hline$u_{0}$ & $0.199 \pm 0.002$ & $-0.202 \pm 0.003$ & $-0.201 \pm 0.005$ & $0.199 \pm 0.003$ & $0.207 \pm 0.004$ \\
\hline$t_{\mathrm{E}}$ (days) & $89.786 \pm 0.232$ & $88.525 \pm 0.316$ & $97.010 \pm 1.345$ & $88.550 \pm 0.308$ & $95.379 \pm 1.024$ \\
\hline$s$ & $1.120 \pm 0.001$ & $1.117 \pm 0.001$ & $1.075 \pm 0.014$ & $1.115 \pm 0.001$ & $1.092 \pm 0.008$ \\
\hline$q$ & $0.632 \pm 0.009$ & $0.664 \pm 0.021$ & $0.724 \pm 0.024$ & $0.648 \pm 0.020$ & $0.736 \pm 0.024$ \\
\hline$\pi_{\mathrm{E}, N}$ & $\cdots$ & $0.033 \pm 0.004$ & $0.382 \pm 0.022$ & $-0.038 \pm 0.003$ & $-0.475 \pm 0.025$ \\
\hline$\pi_{\mathrm{E}, E}$ & $\cdots$ & $0.013 \pm 0.009$ & $0.057 \pm 0.011$ & $0.014 \pm 0.009$ & $-0.026 \pm 0.011$ \\
\hline$d s / d t\left(\mathrm{yr}^{-1}\right)$ & $\ldots$ & $\ldots$ & $0.287 \pm 0.120$ & $\ldots$ & $0.090 \pm 0.067$ \\
\hline$d \alpha / d t\left(\operatorname{rad} \mathrm{yr}^{-1}\right)$ & $\cdots$ & $\cdots$ & $-1.437 \pm 0.138$ & $\cdots$ & $1.574 \pm 0.121$ \\
\hline$F_{\mathrm{S}, \text { OGLE }}$ & $0.248 \pm 0.001$ & $0.251 \pm 0.001$ & $0.252 \pm 0.001$ & $0.250 \pm 0.001$ & $0.253 \pm 0.001$ \\
\hline
\end{tabular}

Note. $H_{J D}^{\prime}=$ HJD - 2450000, Abbreviations-STD: the standard, static model; PRX: the model considering the annual microlens parallax; OBT: the model considering the lens-orbital motion.

fits with $\Delta \chi^{2} \sim 36.4$. However, there do not exist plausible local minima of the $(+,-)$ and $(-,+)$ solutions. ${ }^{20}$

The observed Spitzer light curve is fragmentary and does not cover the caustic-crossing parts. Thus, we expect that "color constraint" might be important for finding the correct model including the Spitzer light curve (described in Section 5.3 of Yee et al. 2015b). To incorporate the color constraint in the modeling, we use $(I-L)_{18}=5.146 \pm 0.124$ as derived in Section 3.3. We then introduce the " $\chi_{\text {penalty }}^{2}$ " defined as

$$
\chi_{\text {penalty }}^{2} \equiv \frac{\left\{2.5 \log \left(F_{\mathrm{S}, \text { Spitzer }} / F_{\mathrm{S}, \mathrm{OGLE}}\right)-(I-L)_{18}\right\}^{2}}{\sigma_{\mathrm{cc}}^{2}},
$$

where $F_{\mathrm{S}, \text { Spizer }}$ and $F_{\mathrm{S} \text {,OGLE }}$ are the source fluxes of each observatory, (i.e., of each passband) determined from the model. The $\sigma_{\mathrm{cc}}$ is the uncertainty of the color constraint. The $\chi_{\text {penalty }}^{2}$ increases as the difference between the model-fitted color and color constraint increases. Note that, we additionally increase the penalty defined as

$$
\begin{gathered}
\left(\chi_{\text {penalty }}^{2}\right)^{\prime}=\operatorname{fac}^{2} \times\left(\chi_{\text {penalty }}^{2}\right), \\
\text { if } 2.5 \log \left(\frac{F_{\mathrm{S}, \text { Spitzer }}}{F_{\mathrm{S}, \text { OGLE }}}\right)> \pm \mathrm{fac} \times \sigma_{\mathrm{cc}}(I-L)_{18},
\end{gathered}
$$

where "fac" is a factor set as 2 . It implies that we use a $\left(\chi_{\text {penalty }}^{2}\right)^{\prime}$ that is four times larger than $\chi_{\text {penalty }}^{2}$ if the color difference is larger than the $2 \sigma$ level of the color constraint.

In Table 2, we present the best-fit parameters of models both with and without the color constraint. The total $\chi^{2}$ consists of the sum of the $\chi^{2}$ for the ground-based data and the $\chi^{2}$ for the Spitzer data; the $\chi^{2}$ from the color constraint is given separately. For the analysis of this event, we find that the color constraint is of minor importance to the model. In fact,

\footnotetext{
${ }^{20}$ For the $(+,-)$ case, we found a model but the $\chi^{2}$ of the model is larger than $\sim 150$ compared to the best-fit model. This $\Delta \chi^{2}$ is too large to claim the $(+,-)$ solution is a degenerate solution because there exist noticeable deviations between observed and model light curves. Thus, the $(+,-)$ model is rejected as one of the degenerate solutions. For the $(-,+)$ case, we could not find any plausible local minima.
}

when fitting without the constraint, the model parameters are recovered within $3 \sigma$ of those of the best-fit model with the constraint. Particularly, the model parameters of the microlens parallax and the lens-orbital effect are recovered within the $2 \sigma$ level. Because the color constraint is both an intrinsic observable and model-independent, the fact that the best-fit models satisfy this constraint serves as an independent check that they correspond to the physical system. Even though the color constraint plays a relatively minor role, we conclude the best-fit models of this event are the cases of models considering the color constraint (bold parameters in Table 2).

\subsection{The Color-Magnitude Diagram Analysis}

The CMD analysis is required for two purposes in this case. First, we obtain the color constraint for the source star for this event. The color constraint is determined by model-independent regression based on the color-color diagram (described in Calchi Novati et al. 2015b). Second, we obtain the value of the angular source radius, $\theta_{*}$, to determine the angular Einstein ring radius, $\theta_{\mathrm{E}}=\theta_{*} / \rho_{*}$. Thanks to good caustic coverage from KMTNet observations, it is possible to securely measure the $\rho_{*}$ value of this event based on the finite source effect. To achieve both purposes requires determining the position of the source on the CMD of the event. The conventional method is to use the $(V-I, I) \mathrm{CMD}$, but this is impossible in this case because the source suffers from severe extinction $\left(A_{I}=4.9\right)$.

We construct an $(I-H, I)$ CMD using data from the OGLE survey and the VISTA Variables and Via Lactea Survey (VVV: Minniti et al. 2010) by cross-matching field stars within $60^{\prime \prime}$ of the source. We use the VVV $H$-band data (taken with a 4 m-class telescope) because the SMARTS observations (taken with a $1.3 \mathrm{~m}$ telescope) do not go deep enough to capture the red giant clump. Based on the CMD (see Figure 3), the centroid of the giant clump, which is the reference to measure the extinction toward the source, is $(I-H, I)_{\text {clump }}=$ $(5.26 \pm 0.03,19.46 \pm 0.05)$. The position of the source on the CMD is determined as follows. First, from the best-fit model (boldface in Table 2), we have $I_{\mathrm{S}, \mathrm{OGLE}}=19.488 \pm 0.004$, where $I_{\mathrm{S}, \text { OGLE }}=18.0-2.5 \log \left(F_{\mathrm{S}, \text { OGLE }}\right) \pm 1.085\left(\sigma_{F_{\mathrm{S}, \text { OGLE }}} / F_{\mathrm{S}, \text { OGLE }}\right)$. Second, based on the SMARTS data, we estimate the source color, 
Table 2

The Best-fit Models of the Combined Observations

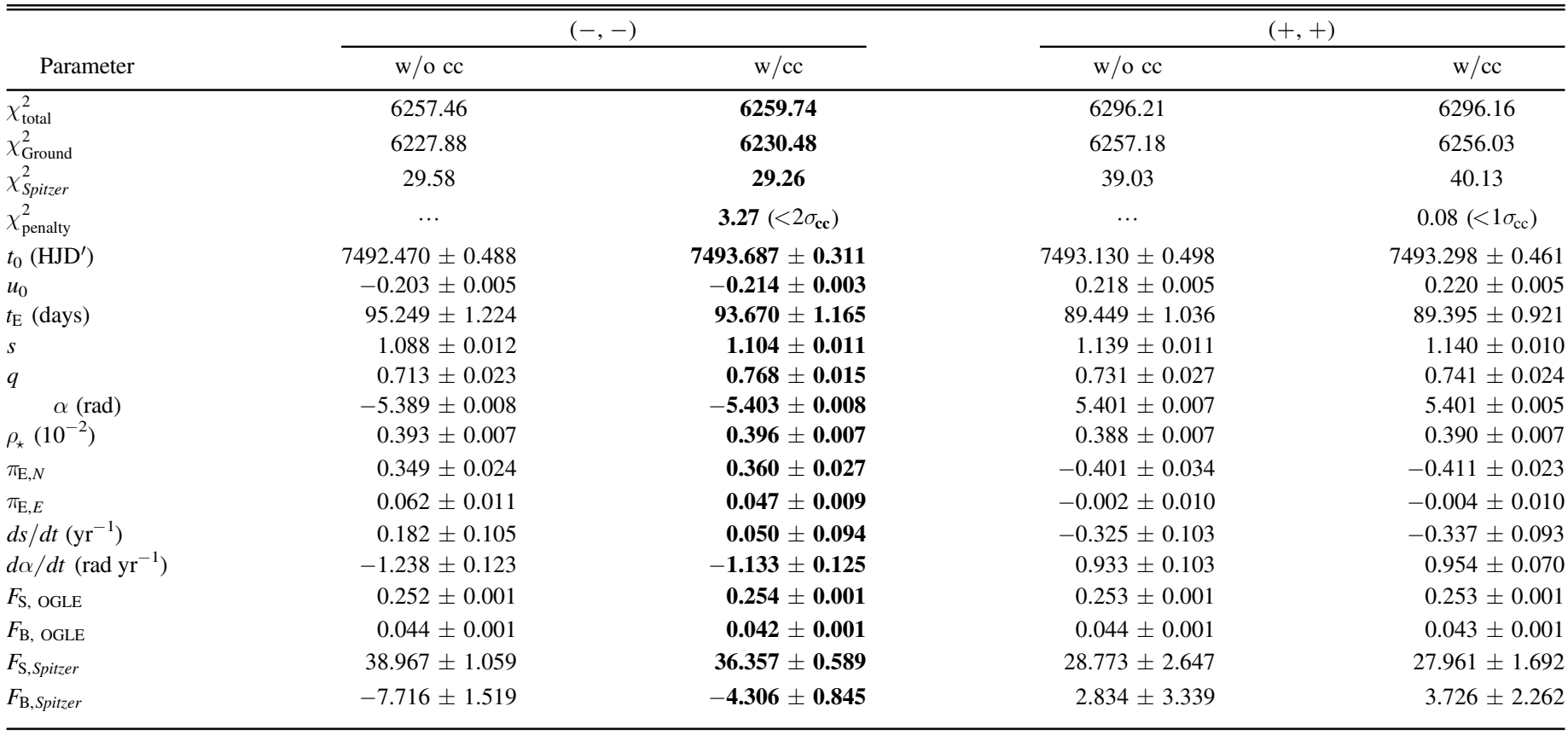

Note. HJD' $=$ HJD -2450000 . See Section 3.3 for the definition of $\chi_{\text {penalty }}^{2}$. The degree of freedom (dof) of each model is $(6260-11)$. The total numbers of groundbased and space-based data that are used for modeling are 6232 and 28 , respectively. Abbreviation—cc: color constraint.

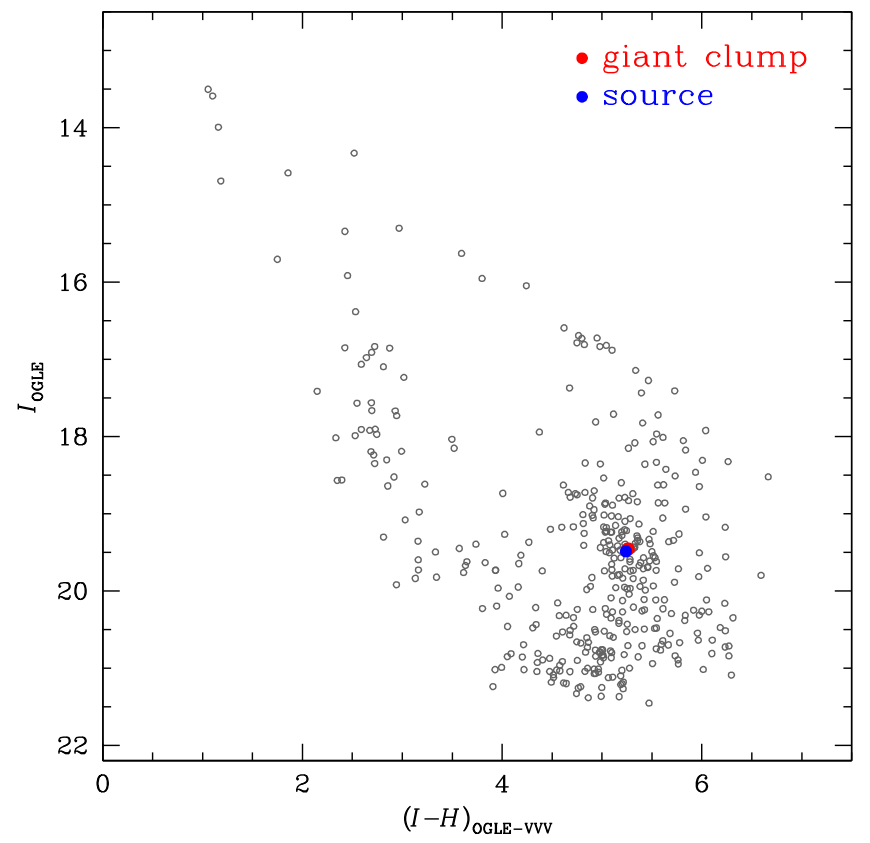

Figure 3. $(I-H, I)$ CMD of the OGLE-2016-BLG-0168 event. The CMD is constructed by cross-matching OGLE and VVV data. The red and blue dots indicate the positions of the centroid of the giant clump and the source star, respectively.

$\left(I_{\mathrm{CTIO}}-H_{\mathrm{CTIO}}\right)_{\mathrm{S}}=2.971 \pm 0.006$, using model-independent regression. We use comparison stars to convert the color to the OGLE and VVV magnitude scale. First, we estimate the difference between OGLE and CTIO, $\left(I_{\mathrm{OGLE}}-I_{\mathrm{CTIO}}\right)=-0.772 \pm$ 0.036. Second, we also estimate the difference between CTIO and $2 \mathrm{MASS},\left(H_{2 \mathrm{MASS}}-H_{\mathrm{CTIO}}\right)=-3.036 \pm 0.014$. Note that the VVV scale is identical to 2MASS. Combining this all together, the location of the source is estimated as $(I-H, I)_{\mathrm{S}}=(5.235 \pm$ $0.039,19.488 \pm 0.004)$. As shown in Figure 3 , the locations of the source and the centroid of the red giant clump are almost identical.

Based on the positions of the source and the centroid of the giant clump on the $(I-H, I) \mathrm{CMD}$, we can apply the conventional method (Yoo et al. 2004) to derive the dereddened color and brightness of the source. First, we convert the color difference $\Delta(I-H) \equiv(I-H)_{\mathrm{S}}-(I-H)_{\text {clump }}$ to $\Delta(V-I) \equiv(V-I)_{\mathrm{S}}-(V-I)_{\text {clump }}$ using the color relations for giants from Bessell \& Brett (1988), which gives $\Delta(V-I)=0.762 \Delta(I-H)$. Second, using the de-reddened $(V-I)$ color and intrinsic $I$ magnitude of the bulge giant clump as a reference (that values adopted from Bensby et al. (2013) and calculated from Nataf et al. (2013), respectively), we determine the de-reddened color and brightness of the source to be $(V-I, I)_{0, \mathrm{~S}}=(1.04 \pm 0.04,14.56 \pm 0.05)$, where $(V-I)_{0, \mathrm{~S}}=(V-I)_{0, \text { clump }}+\Delta(V-I)$ and $I_{0, \mathrm{~S}}=$ $I_{\mathrm{S}}+\left(I_{0, \text { clump }}-I_{\text {clump }}\right)$, which also gives $A_{I}=4.9$. We note that the distance of the source star is estimated by the relation of Nataf et al. (2013), $D_{\mathrm{S}}=D_{\mathrm{GC}} /(\cos l+\sin l \times \cot \phi) \sim$ $8.5 \mathrm{kpc}$, where $D_{\mathrm{GC}}=8160 \mathrm{pc}$ indicates the distance to the galactic center, $l$ is the longitude of the source star in galactic coordinates, and $\phi=40^{\circ}$ is an apparent bar viewing angle, respectively. Then, the angular source radius, $\theta_{*}=5.56 \pm$ $0.45 \mu \mathrm{as}$, is determined by converting $(V-I)$ to $(V-K)$ based on the color-color relation in Bessell \& Brett (1988), and then the angular radius of the source is determined using the color/surface-brightness relation in Kervella et al. (2004). From the value of $\rho_{*}$ derived from the best-fit model, we 
determine that the angular Einstein ring radius of this event is

$$
\theta_{\mathrm{E}}=1.41 \pm 0.12 \text { mas. }
$$

In addition, based on the location on the CMD and the intrinsic color of the source, the source spectral type is estimated to be an early K-type giant. We adopt limb-darkening coefficients appropriate for this source spectral type derived from (Claret 2000). The coefficient for the $I$-band is equal to $\Gamma_{I}=(2 u /(3-u))=0.5103$, where $u_{I}=0.6098$, assuming typical properties of an early K-type giant: effective temperature, $T_{\text {eff }} \sim 4750 \mathrm{~K}$, metallicity, $[M / H] \sim 0.0$, turbulence velocity, $V_{\mathrm{t}}<2.0 \mathrm{~km} \mathrm{~s}^{-1}$, and surface gravity, $\log g \sim 2.0$.

For the color constraint, we carry out color-color regression based on the HIL color-color diagram. The relation is $(I-L)=1.238(I-H)-8.341$, where $I, H$, and $L$ indicate the passbands of OGLE, VVV, and Spitzer, respectively. By adopting the color of the source, $(I-H)_{\mathrm{S}}=5.24$, the color constraint is $(I-L)_{25}=-1.854 \pm 0.124$. We note that this transformation introduces an uncertainty of $0.1 \mathrm{mag}$. We also note that the arbitrary flux system of the Spitzer data has 1 flux unit equal to 25 th magnitude. This system is different from the other observations, which use 1 flux unit equal to 18th magnitude, which again is an arbitrary system but one that is frequently used. Thus, we convert the color constraint from the 25 th magnitude system to the 18th magnitude system by adding 7 magnitudes. Hence, we use $(I-L)_{18}=5.146 \pm$ 0.124 for the constraint.

Although the formal uncertainty in the blended flux as given in Table 2 is small, the blended flux is not meaningfully constrained. The uncertainty for $F_{\mathrm{B}}$, OGLE given in Table 2 assumes that the baseline flux is known to infinite precision. However, the baseline object in the OGLE catalog has $I_{\text {base }}=19.318 \pm 0.092$. Although, $F_{\mathrm{S}}$, OGLE is known very well from the light curve, the value of $F_{\mathrm{B}}$, OGLE must be derived from $F_{\mathrm{B}}=F_{\text {base }}-F_{\mathrm{S}}$. Thus, the true uncertainty in $F_{\mathrm{B}, \mathrm{OGLE}}$ is \pm 0.03 (not 0.001 ), i.e., $F_{\mathrm{B}}$, OGLE is consistent with zero.

\section{Results}

\subsection{The Microlens Parallax Test Based on the Spitzer Observations}

Based on space-based observations, it is possible to conduct a test for verifying the measurement of the annual microlens parallax. In addition, as pointed out by Han et al. (2016a, 2016 b), space-based observations can also provide an opportunity to resolve degenerate solutions. Thus, we conduct a test based on the Spitzer observations to verify the annual microlens parallax and lens-orbital motion effects from results of the ground-based models. In addition, we try to resolve degenerate solutions using the Spitzer observations.

In Figure 2, the red lines indicate the predicted source trajectories that should be seen by Spitzer. These predicted source trajectories are produced using ground-based models considering the annual microlens parallax and lens-orbital effects. By comparing the prediction without Spitzer and the fitting with Spitzer data, it is possible to check the measurement of the microlens parallax and lens-orbital motion. Note that the parameters of the annual and satellite microlens parallaxes are defined in the same reference frame (Calchi Novati et al. 2015a; Yee et al. 2015b; Zhu et al. 2015). As a result, it is possible to directly compare the microlens parallax values. In addition, this validation process can provide a chance to resolve the degenerate solutions.

As shown in Figure 2 (purple dots on the predicted trajectories), Spitzer observations covered only a short segment ( $\sim 26$ days) compared to the total Einstein timescale of the event ( $\sim 94$ days). For relatively long-timescale microlensing events, space-based observations usually cover only part of the lensing light curve due to the short observing window. As a result, this fragmentary Spitzer light curve is quite common for long-timescale lensing events. Thus, our test can provide an important example of whether it is possible to extract secure microlens parallax information from a fragmentary light curve or not.

\subsection{Breaking the Degeneracy of the Microlens Parallax}

For the case of the OGLE-2016-BLG-0168 event, we found two possible lens-parallax solutions, $(-,-)$ and $(+,+)$, out of the possible four-fold degeneracy for the microlens parallax. The $\Delta \chi^{2}$ between those models is $\sim 36.4,10.9$ of which comes from $\Delta \chi_{\text {Spitzer }}^{2}$. In addition, we found inconsistency between the prediction of the light curve covered by the Spitzer data made from the ground-based data alone for the $\left(u_{0}>0\right)$ solution and the best-fit model including Spitzer data for the $(+,+)$ solution, as indicated by the different curvatures of the Spitzer light curve seen in Figure 2. Furthermore, for the prediction of the $(+,+)$ case, there exist large inconsistencies in the parameters between the $\left(u_{0}>0\right)$ ground-only model and the model including Spitzer data for the $(+,+)$ case at the $4 \sigma$ and more than $6 \sigma$ levels for the microlens parallax and lensorbital parameters, respectively (see Figure 4). Hence, considering all the clues to resolve the degeneracy, we conclude the $(-,-)$ model is the unique solution that describes the nature of the binary lens system of this lensing event.

\subsection{Confirmation of the Annual Microlens Parallax}

As shown in Figure 2, for the $(-,-)$ case, the prediction of the Spitzer light curve is almost the same as the light curve found by including the Spitzer data in the fitting. Thus, the higher-order effects measured from the ground-based light curve alone are confirmed by the Spitzer observations. Note that the prediction of the space-based light curve is dominated by the microlens parallax parameters. However, the microlens parallax parameters are strongly affected by the lens-orbital effect (Shin et al. 2012). Thus, the lens-orbital parameters are also essential factors for the successful prediction of the Spitzer light curve.

In Figure 4, we present distributions of the microlens parallax and lens-orbital parameters to clearly show the confirmation of the prediction for the $\left((-,-)\right.$ and $\left.\left(u_{0}<0\right)\right)$ case. We find that the parameters of the ground $\left(u_{0}<0\right)$ model that are used for the prediction are well matched to those of the model including Spitzer data for the $(-,-)$ case, i.e., within $2 \sigma$ and $3 \sigma$ for the microlens parallax and lens-orbital parameters, respectively.

\subsection{Value of Fragmentary Spitzer Observations}

The confirmation of the microlens parallax and the resolution of the $(-,-)$ to $(+,+)$ degeneracy show that it is possible to extract valuable information from space-based observations even though the observations are fragmentary. Although this is one 
w/ Spitzer w/o Spitzer

$(-,-)$ case $\left(u_{0}<0\right)$
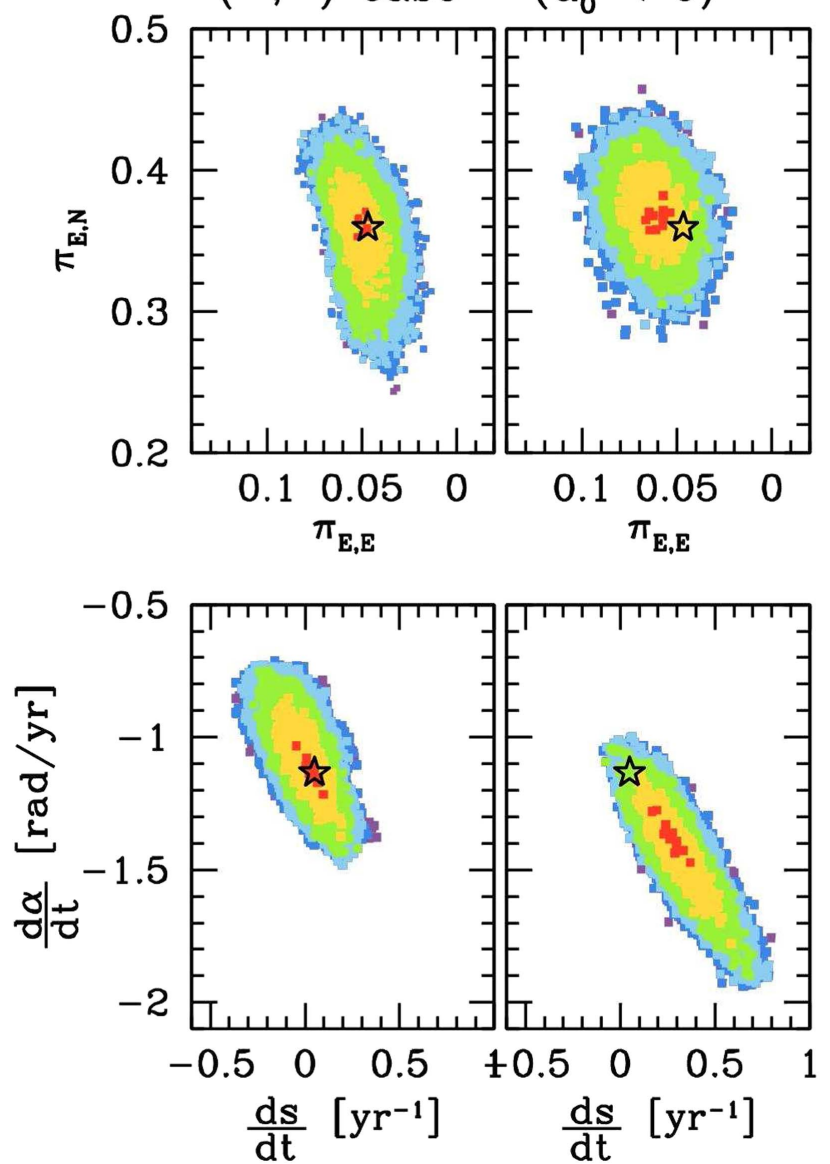

w/ Spitzer w/o Spitzer
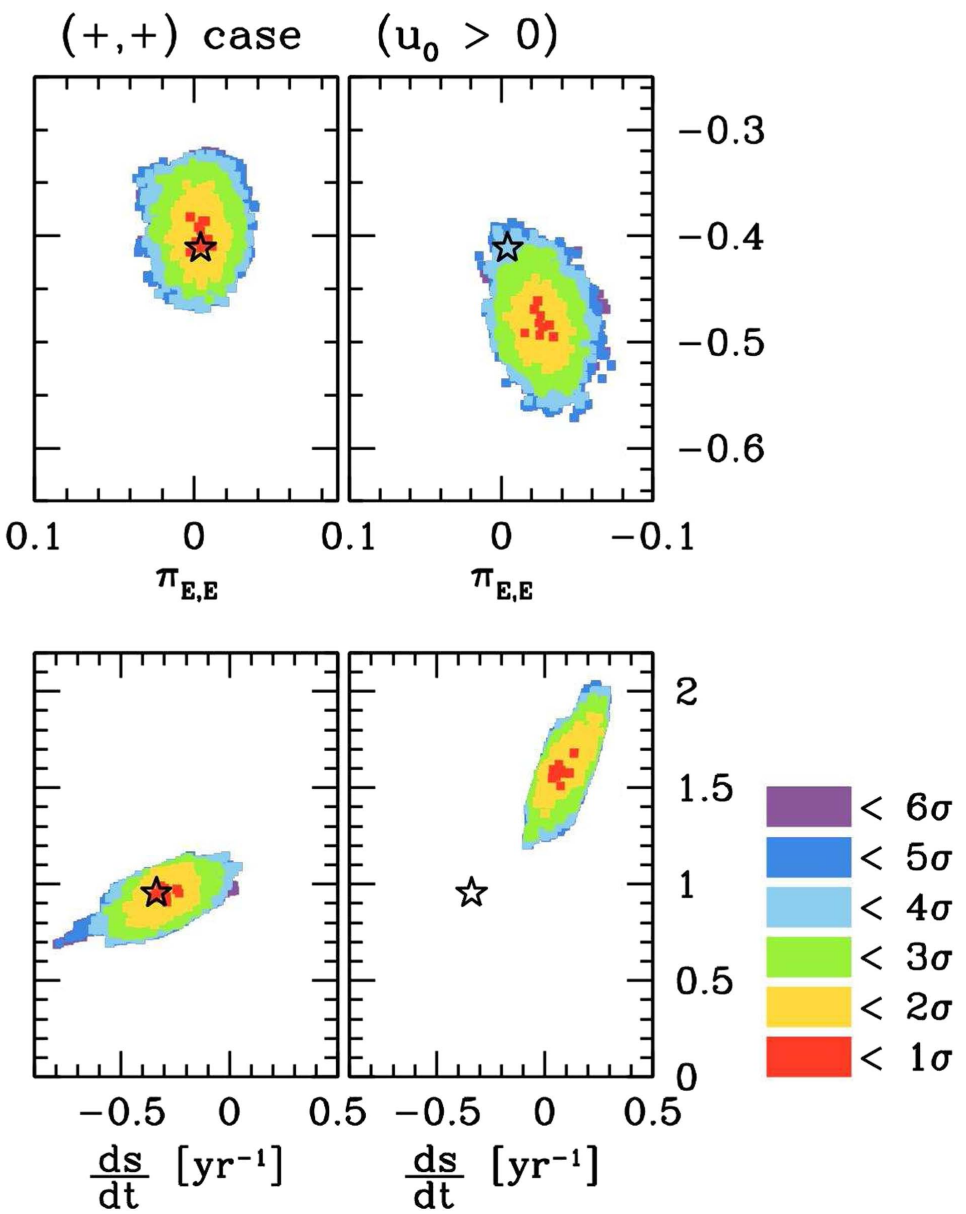

Figure 4. The distributions of the microlens parallax and lens-orbital motion parameters. The left panels show $(-,-)$ and $\left(u_{0}<0\right)$ cases of the MCMC chain scatter with and without the Spitzer data. Red, yellow, green cyan, blue, and purple represent the $\Delta \chi^{2}$ between the best-fit and chain values of less than 1, 2, 3, 4, 5, and $6 \sigma$, respectively. The star represents the best-fit value of the model including the Spitzer data. The right panels show $(+,+)$ and $\left(u_{0}>0\right)$ cases with an identical scheme to the left panels.

specific case, it is significant because almost all space-based observations have only partial coverage of long-timescale lensing events. Thus, we frequently encounter such fragmentary light curves.

\subsection{Properties of the Binary Lens System}

Based on the unique best-fit model and combining the information of the microlens parallax and the angular Einstein ring radius, we can determine the properties of the binary lens system according to Equations (1). In Table 3, we present the properties of the lens system. The system consists of nearly equal mass stars,

$$
M_{1}=0.27 \pm 0.03 M_{\odot} ; M_{2}=0.21 \pm 0.02 M_{\odot},
$$

with a projected separation,

$$
a_{\perp}=2.47 \pm 0.23 \mathrm{au} .
$$

The lens system is located $1.59 \pm 0.15 \mathrm{kpc}$ from us. We check the possibility of follow-up observations to confirm the properties of the lens system. Considering the relative lens-source proper

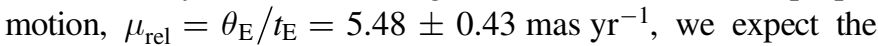
binary lens and source star to separate by $\sim 55$ mas after 10 years from now. Given that JWST's resolution is $\sim 70$ mas, JWST will
Table 3

The Properties of the Binary Lens System

\begin{tabular}{lc}
\hline \hline Quantity & Value \\
\hline Einstein radius, $\theta_{\mathrm{E}}(\mathrm{mas})$ & $1.41 \pm 0.12$ \\
Total Mass, $M_{\mathrm{total}}\left(M_{\odot}\right)$ & $0.48 \pm 0.05$ \\
Primary Mass, $M_{1}\left(M_{\odot}\right)$ & $0.27 \pm 0.03$ \\
Secondary Mass, $M_{2}\left(M_{\odot}\right)$ & $0.21 \pm 0.02$ \\
Distance to lens, $D_{\mathrm{L}}(\mathrm{kpc})$ & $1.59 \pm 0.15$ \\
Projected separation, $a_{\perp}(\mathrm{au})$ & $2.47 \pm 0.23$ \\
Geocentric proper motion, $\mu_{\mathrm{geo}}\left(\mathrm{mas} \mathrm{yr}^{-1}\right)$ & $5.48 \pm 0.45$ \\
Heliocentric proper motion, $\mu_{\text {hel }}\left(\mathrm{mas} \mathrm{yr}^{-1}\right)$ & $6.21 \pm 0.51$ \\
Stability of system, KE $/ \mathrm{PE}^{\mathrm{a}}$ & 0.52
\end{tabular}

Note.

${ }^{\mathrm{a}}$ If the ratio of the kinetic to potential energy of the binary lens system (KE/PE) is less then 1 , then the orbital motion of binary components is physically allowed. However, values $(\mathrm{KE} / \mathrm{PE}) \sim 1$ and values $(\mathrm{KE} / \mathrm{PE}) \ll 1$ would require very special physical configurations and/or viewing angles. Hence, the parameters of the model considering the lens-orbital effect are quite reasonable values.

have difficulty making follow-up observations. However, using next-generation telescopes (30 m-class), it is quite possible that the lens will be visible for follow-up observations. 


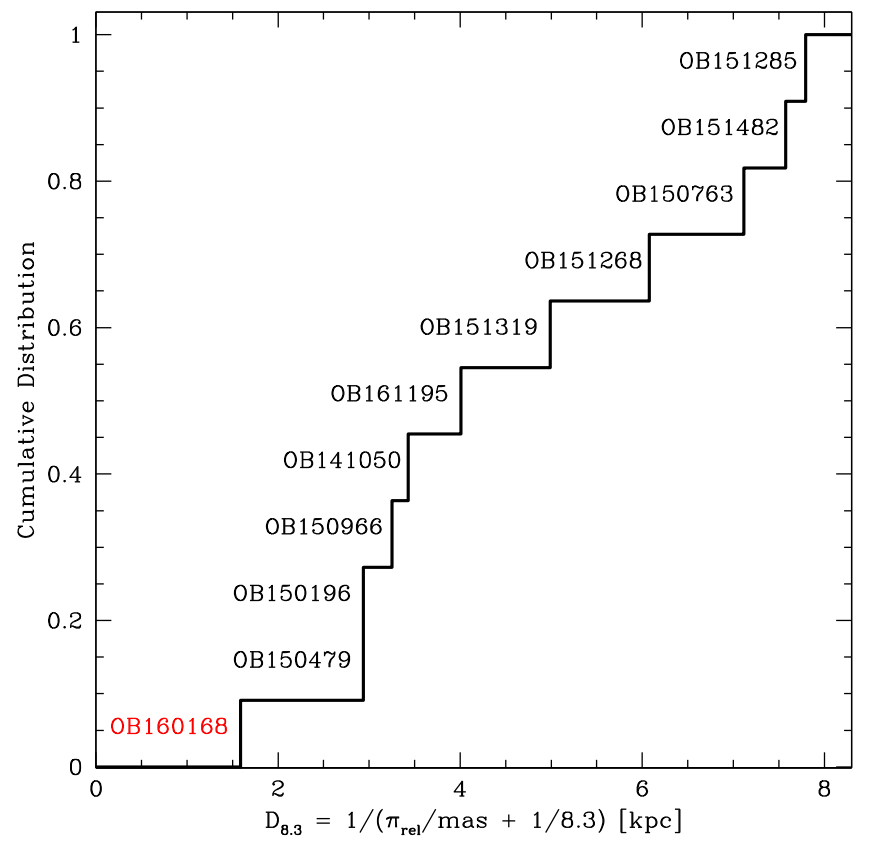

Figure 5. Cumulative distribution of $D_{8.3}$ of Spitzer microlensing events. We adopted the $\pi_{\text {rel }}$ value from the published result of each case: OB141050 (Zhu et al. 2015), OB150196 (Han et al. 2017), OB150479 (Han et al. 2016a), OB150763 (Zhu et al. 2016), OB150966 (Street et al. 2016), OB151268 (Zhu et al. 2016), OB151285 (Shvartzvald et al. 2015) OB151319 (Shvartzvald et al. 2016), OB151482 (Chung et al. 2017), and OB161195 (Shvartzvald et al. 2017).

Since we introduce orbital motion of the lens system, we check whether the best-fit orbital parameters are physically reasonable or not. Thus, we derive the ratio of kinetic to potential energy of the system to validate the stability of the lens system. The determined value $(\mathrm{KE} / \mathrm{PE}) \simeq 0.5$ easily satisfies the physically bound condition $(\mathrm{KE} / \mathrm{PE})<1$. Moreover, it is well away from the regimes $(\mathrm{KE} / \mathrm{PE}) \sim 1$ and $(\mathrm{KE} / \mathrm{PE}) \ll 1$, both of which would require special geometries and/or viewing angles. Since the orbital motion parameters are derived from quite subtle features in the light curve, they could, in principle, reflect unrecognized systematic errors in the data rather the the physical dynamics of the system. However, since the former putative explanation can yield arbitrarily high or low values of $(\mathrm{KE} / \mathrm{PE})$, whereas the latter explanation must yield $(\mathrm{KE} / \mathrm{PE})<1$, and will yield $0.1<(\mathrm{KE} / \mathrm{PE})<0.8$ for the great majority of binaries, the fact that the measured value is near the middle of this range can be regarded as evidence that binary motion, rather than systematics, is the true origin. This evidence is relatively weak for any individual case, but if the test is successfully repeated for an ensemble of events, the evidence will be much stronger. This is important in the present case because the lens is unusually close $\left(D_{L}=1.6 \mathrm{kpc}\right)$ and the orbital motion is usually fast $\left(|d \alpha / d t| \sim 1 \operatorname{radian} \mathrm{yr}^{-1}\right)$. A number of the most interesting microlensing events, e.g., OGLE-2011-BLG-0417 (Shin et al. 2012), OGLE-2011-BLG0420 and OGLE-2009-BLG-151 (Choi et al. 2013), are from such nearby lenses, which are intrinsically relatively rare but which frequently permit ground-based parallax measurements when they occur. Hence, when one of these can be verified as a physically (rather than systematics) generated light curve by several independent checks, it enhances confidence in this entire interesting class of events.
In Figure 5, we present the cumulative distribution of the "distance parameter," $D_{8.3}$ (see Section 5 of Calchi Novati et al. 2015a), of published microlensing events with wellmeasured $\pi_{\mathrm{rel}}\left(=\pi_{\mathrm{E}} \theta_{\mathrm{E}}\right)$ based on Spitzer observations. We note that the lens system of this work is the nearest one with a Spitzer distance. Assuming that two-body lenses, which dominate this sample, follow the same galactic distribution as all lenses, this distribution represents the most precise determination of the Spitzer-observed lens distance distribution. By comparing this precise distance distribution of all stellar objects to those of planets, it is possible to study differences in the planet distance distribution. Thus, this distribution is a key factor in understanding the distribution of planets in our galaxy.

\section{Summary and Discussion}

We analyzed the microlensing event OGLE-2016-BLG-0168 based on combined ground- and space-based observations obtained from the OGLE, KMTNet, and Spitzer telescopes. It is possible to clearly detect signals of higher-order effects in the light curve that are caused by the finite source effect, the microlens parallax, and the orbital motion of the binary lens components. Based on the additional information from these high-order effects, we found that this event was created by a binary system consisting of almost equal mass M-dwarf stars $\left(\sim 0.27\right.$ and $\left.\sim 0.21 M_{\odot}\right)$ with a projected separation $\sim 2.5$ au. The system is located $\sim 1.6 \mathrm{kpc}$ from us.

We successfully predict the Spitzer light curve of the $(-,-)$ model case based on the annual microlens parallax measured using the ground-based observations. The annual microlens parallax is confirmed at the $2 \sigma$ level by the satellite microlens parallax measured with Spitzer observations. In addition, it is possible to resolve the degenerate solutions using the Spitzer observations.

Our test of the microlens parallax can provide an important example for preparing for the new era of the microlensing technique in collaboration with space-based observations. In principle, the microlensing technique can detect a variety of astronomical objects regardless of their brightnesses. However, additional observables are required to reveal what kind of object produced the microlensing event. Among these essential observables, the microlens parallax is one of the key pieces of information that reveals the nature of the lens of the event. Thus, it is important to routinely and securely measure the microlens parallax. Before collaborations with space-based observations, measuring the microlens parallax usually depended on the timescale of the lensing event. For some lensing events with long timescales, the microlens parallax signal can be detected. However, this annual microlens parallax might be inaccurately measured due to systematics in the data. With the commencement of the era of space-based observation collaborations, however, the microlens parallax can be routinely measured regardless of the timescale and magnification level of the lensing event.

Since most space-based observations cover only part of the full lensing light curves with a long timescale due to the relatively short observing window, it is important to conduct a test to determine whether or not it is possible to extract secure information on the microlens parallax. In addition, since spacebased observations can provide a chance to resolve degenerate solutions, it is also important to conduct another test to 
determine whether or not the degeneracy breaking is possible using fragmentary space-based observations.

We conduct the microlens parallax test using the fragmentary Spitzer observation of the OGLE-2016-BLG-0168 binary lensing event. Our test result provides an example showing that it is possible to verify the microlens parallax and resolve the degeneracy based on space-based observations, even though the observation is fragmentary. This result gives confidence to other collaborations that will measure the microlens parallax between next-generation microlensing surveys and space telescopes such as the Spitzer microlensing campaign (Yee et al. 2015a), K2C9 (Henderson et al. 2016), and WFIRST (Spergel et al. 2015).

This research has made use of the KMTNet system operated by the Korea Astronomy and Space Science Institute (KASI) and the data were obtained at the three host sites of CTIO in Chile, SAAO in South Africa, and SSO in Australia. This work is based in part on observations made with the Spitzer Space Telescope, which is operated by the Jet Propulsion Laboratory, California Institute of Technology under a contract with NASA. The OGLE project has received funding from the National Science Centre, Poland, grant MAESTRO 2014/14/A/ST9/ 00121 to A. Udalski. Work by I-G. Shin and A. Gould was supported by JPL grant 1500811. A. Gould, Y. K. Jung, and W. Zhu acknowledge the support from NSF grant AST-1516842. Work by C. Han was supported by the Creative Research Initiative Program (2009-0081561) of the National Research Foundation of Korea. Work by Y.S. and C.B.H. was supported by an appointment to the NASA Postdoctoral Program at the Jet Propulsion Laboratory, administered by Universities Space Research Association through a contract with NASA.

\section{ORCID iDs}

I.-G. Shin (i) https://orcid.org/0000-0002-4355-9838

J. C. Yee (iD https://orcid.org/0000-0001-9481-7123

S. Calchi Novati (i) https://orcid.org/0000-0002-7669-1069

J. Skowron (iD https://orcid.org/0000-0002-2335-1730

S. Kozłowski ib https://orcid.org/0000-0003-4084-880X

P. Pietrukowicz (i) https://orcid.org/0000-0002-2339-5899

M. D. Albrow (iD https://orcid.org/0000-0003-3316-4012

S.-J. Chung (iD https:// orcid.org/0000-0001-6285-4528

K.-H. Hwang (ib https://orcid.org/0000-0002-9241-4117

Y. K. Jung (i) https://orcid.org/0000-0002-0314-6000

Y.-H. Ryu (iD https://orcid.org/0000-0001-9823-2907

W. Zhu (iD https://orcid.org/0000-0003-4027-4711

R. W. Pogge (iD https://orcid.org/0000-0003-1435-3053

S. Carey (i) https://orcid.org/0000-0002-0221-6871

B. S. Gaudi (1) https://orcid.org/0000-0003-0395-9869

C. B. Henderson (iD https://orcid.org/0000-0001-8877-9060

Y. Shvartzvald (iD https://orcid.org/0000-0003-1525-5041

\section{References}

Alard, C. , \& Lupton, R. H. 1998, ApJ, 503, 325

Albrow, M. D., Horne, K., Bramich, D. M., et al. 2009, MNRAS, 397, 2099

An, J. H. 2005, MNRAS, 356, 1409

Batista, V., Gould, A., Dieters, S., et al. 2011, A\&A, 529, A102

Bensby, T., Yee, J. C., Feltzing, S., et al. 2013, A\&A, 549, 147

Bessell, M. S., \& Brett, J. M. 1988, PASP, 100, 1134

Calchi Novati, S., Gould, A., Udalski, A., et al. 2015a, ApJ, 804, 20

Calchi Novati, S., Gould, A., Yee, J. C., et al. 2015b, ApJ, 814, 92

Cardelli, J. A., Clayton, G. C., \& Mathis, J. S. 1989, ApJ, 345, 245

Choi, J.-Y., Han, C., Udalski, A., et al. 2013, ApJ, 768, 129

Chung, S.-J., Zhu, W., Udalski, A., et al. 2017, ApJ, 838, 154

Claret, A. 2000, A\&A, 363, 1081

Dominik, M. 1999, A\&A, 349, 108

Dong, S., Udalski, A., Gould, A., et al. 2007, ApJ, 664, 862

Gould, A. 1992, ApJ, 392, 442

Gould, A. 1994, ApJ, 421, 71

Graff, D. S., \& Gould, A. 2002, ApJ, 580, 253

Griest, K., \& Safizadeh, N. 1998, ApJ, 500, 37

Han, C., Udalski, A., Gould, A, et al. 2016a, ApJ, 828, 53

Han, C., Udalski, A., Gould, A., et al. 2017, ApJ, 834, 82

Han, C., Udalski, A., Lee, C.-U., et al. 2016b, ApJ, 827, 11

Henderson, C. B., Poleski, R., Penny, M., et al. 2016, PASP, 128, 124401

Hirao, Y., Udalski, A., Sumi, T., et al. 2017, AJ, 154, 1

Jiang, G., DePoy, D. L., Gal-Yam, A., et al. 2004, ApJ, 617, 1307

Jung, Y. K., Udalski, A., Sumi, T., et al. 2015, ApJ, 798, 123

Kervella, P., Bersier, D., Mourard, D., et al. 2004, A\&A, 428, 587

Kim, S.-L., Lee, C.-U., Park, B.-G., et al. 2016, JKAS, 49, 37

Minniti, D., Lucas, P. W., Emerson, J. P., et al. 2010, NewA, 15, 433

Miyake, N., Udalski, A., Sumi, T., et al. 2012, ApJ, 752, 82

Nataf, D. M., Gould, A., Fouqué, P., et al. 2013, ApJ, 769, 88

Poindexter, S., Afonso, C., Bennett, D. P., et al. 2005, ApJ, 633, 914

Poleski, R., Zhu, W., Christie, G. W., et al. 2016, ApJ, 823, 63

Refsdal, S. 1966, MNRAS, 134, 315

Shin, I.-G., Han, C., Choi, J.-Y., et al. 2012, ApJ, 755, 91

Shin, I.-G., Sumi, T., Udalski, A., et al. 2013, ApJ, 764, 64

Shin, I.-G., Udalski, A., Han, C., et al. 2011, ApJ, 735, 85

Shvartzvald, Y., Li, Z., Udalski, A., et al. 2016, ApJ, 831, 183

Shvartzvald, Y., Udalski, A., Gould, A., et al. 2015, ApJ, 814, 111

Shvartzvald, Y., Yee, J. C., Calchi Novati, S., et al. 2017, ApJL, 840, L3

Skowron, J., Udalski, A., Gould, A., et al. 2011, ApJ, 738, 87

Skowron, J., Udalski, A., Kozłowski, S., et al. 2016, AcA, 66, 1

Smith, M. C., Mao, S., \& Paczyński, B. 2003, MNRAS, 339, 925

Spergel, D., Gehrels, N., Baltay, C., et al. 2015, arXiv:1503.03757

Street, R. A., Udalski, A., Calchi Novati, S., et al. 2016, ApJ, 819, 93

Udalski, A. 2003, AcA, 53, 291

Udalski, A., Szymański, M., Kaluzny, J., et al. 1994, AcA, 44, 227

Udalski, A., Szymański, M. K., \& Szymański, G. 2015, AcA, 65, 1

Udalski, A., Yee, J. C., Gould, A., et al. 2015, ApJ, 799, 237

Wozniak, P. R. 2000, AcA, 50, 421

Wyrzykowski, Ł., Kostrzewa-Rutkowska, Z., Skowron, J., et al. 2016, MNRAS, 458, 3012

Yee, J. C., Gould, A., Beichman, C., et al. 2015a, ApJ, 810, 155

Yee, J. C., Udalski, A., Calchi Novati, S., et al. 2015b, ApJ, 802, 76

Yoo, Jaiyul., DePoy, D. L., Gal-Yam, A., et al. 2004, ApJ, 603, 139

Zhu, Wei., Calchi Novati, S., Gould, A., et al. 2016, ApJ, 825, 60

Zhu, Wei., Udalski, A., Calchi Novati, S., et al. 2017, ApJ, submitted (arXiv:1701.05191)

Zhu, Wei, Udalski, A., Gould, A., et al. 2015, ApJ, 805, 8 